\title{
An Unusual Case of Cholestatic Hepatitis due to Light-Chain Deposition Disease
}

\author{
Alessandro Grembiale ${ }^{a} \quad$ Elena Garlattia Anna Ermacora ${ }^{a} \quad$ Silvia Graziolia \\ Massimiliano Balbi ${ }^{b}$ Maurizio Tonizzo ${ }^{a}$ \\ aDepartment of Internal Medicine, ASFO - Pordenone, Pordenone, Italy; bepartment of \\ Internal Medicine, ASFO - San Vito al Tagliamento (PN), San Vito al Tagliamento, Italy
}

\section{Keywords}

Light-chain deposition disease · Monoclonal light chains - Amyloidosis - Cholestatic hepatitis . Bortezomib

\begin{abstract}
Light-chain deposition disease (LCDD) is a rare paraproteinaemia characterized by the deposition of monoclonal immunoglobulins with a non-fibrillar structure and hence Congo red negative deposits. Kidney disease is the more frequent manifestation, but other organs may also be involved. A 70-year-old man with hypertension and mild chronic renal failure showed a hepatomegaly without splenomegaly. His renal and liver test rapidly got worse. A serum electrophoresis and immunofixation isolated monoclonal kappa light-chain gammopathy, with serum free kappa light chain excess. The bone marrow biopsy showed the presence of interstitial infiltration of plasma cells like multiple myeloma type at initial phase. Periumbilical fat biopsy was negative. Echocardiography demonstrated an infiltrative cardiac disease. The biopsies of the duodenum small intestine mucosa showed flaps with eosinophil material (Masson's staining) with atrophic crypts and chronic inflammation at chorion level. Amyloid substance was negative. There was a strong positivity for light chains kappa compatible with LCDD. A liver biopsy confirmed this finding. Therapy with dexamethasone and bortezomib improved clinical state and hepatic and renal laboratory tests. Chemotherapy based on novel anti-myeloma agents should be rapidly considered in LCDD patients with severe organ involvement.




\section{Introduction}

Light-chain deposition disease (LCDD), heavy-chain deposition disease, and light- and heavy-chain deposition disease are a rare group of paraproteinaemias characterized by the deposition of monoclonal immunoglobulins with a non-fibrillar structure and hence Congo red negative deposits [1].

The diagnosis of LCDD requires histological demonstration of monotypic light-chain (LC) deposition on immunofluorescence microscopy and ultrastructural analysis of the involved organs or tissues. LCDD can occur in the context of isolated monoclonal gammopathy or of symptomatic multiple myeloma and Waldenström's macroglobulinemia. Light chain deposits are usually the $\kappa$ (kappa) isotype and can affect almost all organs [2]. Kidney disease is the more frequent manifestation, resulting in chronic kidney failure with glomerular proteinuria, and sometimes nephrotic syndrome [3] but heart, liver [4], gastrointestinal tract, and peripheral nerves may also be involved. Liver involvement has been rarely reported in LCDD in asymptomatic patients, but in symptomatic ones, LCDD-associated liver involvement mainly manifests as cholestatic hepatitis and is associated with high mortality [5].

We report in this paper a patient with myeloma-associated LCDD who developed rapidly progressive liver and renal failure secondary to $\kappa$-light chain deposition, which rapidly recovered after chemotherapy. Patient has given his written informed consent to publish his case.

\section{Case Report}

A 70-year-old man with hypertension, kidney stones disease and mild chronic renal failure was admitted to our department with asthenia and sudden weight loss. Physical examinations showed hepatomegaly without splenomegaly. A liver ultrasound confirmed hepatomegaly with mild hepatic steatosis and a non-homogeneous echostructure with a starry sky appearance. There was no evidence of biliary obstruction, and the kidneys had a normal size without urinary tract obstruction. There was liver stiffness (Fibroscan ${ }^{\odot}: 53.3 \mathrm{kPa}$ with IRQ 18).

Blood tests showed serum creatinine: $2.3 \mathrm{mg} / \mathrm{dL}$, ESR: $120 \mathrm{~mm} / \mathrm{h}, \gamma \mathrm{GT}: 2003 \mathrm{IU} / \mathrm{L}, \mathrm{P}-\mathrm{ALC}$ : $732 \mathrm{IU} / \mathrm{L}$, fibrinogen: $700 \mathrm{mg} / \mathrm{dL}$, presence of monoclonal component IgA k: $14 \mathrm{~g} / \mathrm{L}$. Baseline liver tests, serum calcium, and blood coagulation parameters were normal. There was no history of alcohol abuse. Serological tests for hepatitis A, B and C, Epstein-Barr virus, cytomegalic virus, and herpes simplex virus were negative.

A few weeks later, renal and liver test rapidly got worse (serum creatinine: $6.7 \mathrm{mg} / \mathrm{dL}$, total bilirubin: $4.8 \mathrm{mg} / \mathrm{dL}$, direct bilirubin: $3.9 \mathrm{mg} / \mathrm{dL}$, AST: $647 \mathrm{U} / \mathrm{L}, \mathrm{ALT}: 485 \mathrm{U} / \mathrm{L}, \mathrm{LDH}: 780$ $\mathrm{U} / \mathrm{L}$ ), probably due to a concomitant septic state.

A serum electrophoresis and immunofixation isolated monoclonal kappa LC gammopathy, with serum free kappa light chain excess of $47 \mathrm{mg} / \mathrm{L}$, with a kappa/lambda ratio of 2,76. 24-h proteinuria was $1.71 \mathrm{~g}$, Bence-Jones proteinuria was negative.

The whole body radiological evaluation did not demonstrate osteolytic lesions. The bone marrow biopsy showed the presence of interstitial infiltration (between 10 and 20\%) of plasma cells like a plasmacellular dyscrasia preferentially multiple myeloma type at initial phase.

Moreover, we performed a periumbilical fat biopsy that was negative for the staining with the Congo red, and there were no aspects related to amyloid deposits.

Transthoracic echocardiography demonstrated moderate hypertrophic cardiomyopathy (no pulmonary hypertension), with mainly septal evidence of infiltrative cardiac disease (left

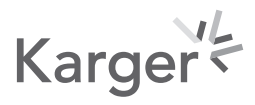




\section{Case Reports in Oncology}

\begin{tabular}{l|l}
\hline Case Rep Oncol 2020;13:1343-1348 \\
\hline DOI: 10.1159/000509508 & $\begin{array}{l}\text { @ 2020 The Author(s). Published by S. Karger AG, Basel } \\
\text { www.karger.com/cro }\end{array}$ \\
\hline
\end{tabular}

Grembiale et al.: A Cholestatic Hepatitis due to LCDD
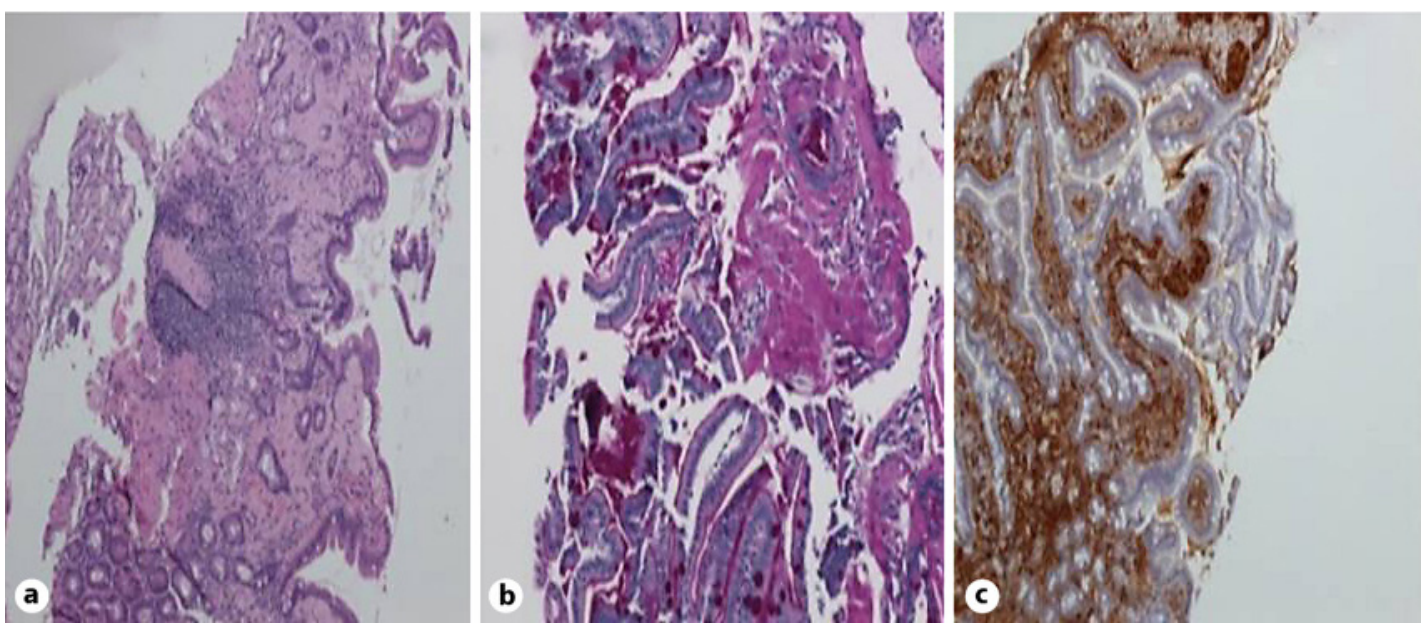

Fig. 1. a, b Duodenum small intestine hematoxylin-eosin staining. c DAB staining.
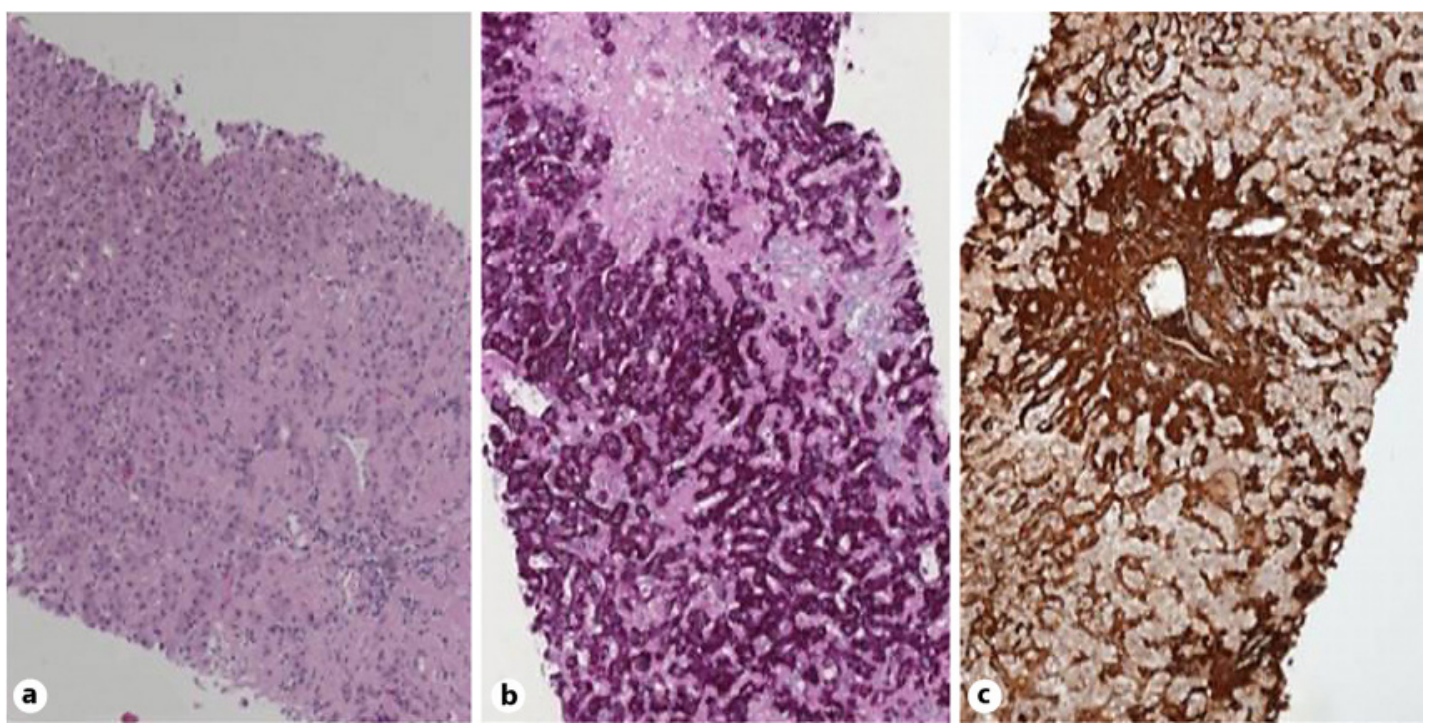

Fig. 2. a, b Liver section hematoxylin-eosin staining. c DAB staining.

ventricle ejection fraction 60\%) and organized pericarditis adherent to the right ventricle (thickness $14 \mathrm{~mm}$ ), without signs of compression on the cardiac chambers.

The patient also underwent gastroscopy, and the biopsies of the duodenum small intestine mucosa showed flaps with eosinophil material at the level of the lamina propria (Masson's staining) with atrophic crypts and chronic inflammation at the chorion level (Fig. 1 a, 1. b, 1. c). The Congo red staining for the research of amyloid substance was negative. The search for amyloid $\mathrm{A}$ and $\mathrm{P}$ was negative; but there was a strong positivity for light chains kappa +++ on immunohistochemistry, preferentially compatible with LCDD.

A liver biopsy was also performed which confirmed the presence of amorphous eosinophilous deposits at the sinusoidal level associated with atrophy moderate hepatic parenchyma (Fig. 2 a, 2. b, 2. c). The substance was Congo red negative, kappa +++ light chains, PAS-. 
We concluded that it was LCDD with hepatic, gastrointestinal, probably renal and cardiac involvement, associated with IgA K myeloma. We started therapy with dexamethasone and bortezomib with improvement of hepatic and renal function laboratory tests.

We observed a progressive and complete recovery of cholestatic and cytolytic hepatitis over the next 6 months with mild improvement of renal failure.

\section{Discussion}

LCDD is a rare disease of non-fibrillar deposition of monoclonal light-chain immunoglobulins, usually manifesting in the fifth or sixth decade of life with a male predominance, but the incidence is unknown [6]. In LCDD, the monoclonal light chains are of the kappa subtype in $92 \%$ of cases, and it is typically associated with multiple myeloma or other lymphoproliferative disorders [6].

LCDD occurs in about $5 \%$ of patients with multiple myeloma, typically reported as the underlying disease in these patients $[7,8]$. However, recent reports indicate that a high proportion of LCDD cases are now found in patients without symptomatic plasma cell disorder, who meet the new criteria for monoclonal gammopathy of renal significance [9]. Less frequently, LCDD may occur in the context of lymphoid hemopathy such as Waldenström disease. Unlike AL amyloidosis, LC deposits in LCDD, mostly the kappa isotype, do not stain with Congo red, and show a typical histological linear pattern along basement membranes on immunofluorescence microscopy, with a characteristic linear powdery punctate appearance on electron microscopy $[10,11]$.

Based on histological and postmortem studies, liver involvement has been considered to be frequent in LCDD, but severe liver complications have been rarely reported [12]. Principal presentation was intrahepatic cholestasis, associated with cytolysis in $33 \%$ of cases. The most frequent histological finding was linear deposits of monoclonal LC in the sinusoidal or perisinusoidal spaces with a predominance of kappa isotype, as we reported.

A cardiac origin was also unlikely to account for liver failure, given the absence of right ventricular heart failure at echocardiography. The transient septic state may have contributed to worsening liver and kidney function, but its resolution and the liver biopsy results undoubtedly showed evidence of LC deposition. We finally attributed liver and kidney failure as a specific manifestation of the disease in our patient.

Steroids and melphalan, high-dose melphalan, autologous stem cell transplantation and, more recently, bortezomib-based chemotherapy, are some of the treatment options [13]. Chemotherapeutic regimens containing the proteasome inhibitor bortezomib have shown efficacy and good tolerance profile in both AL amyloidosis [14] and Randall-type MIDD, but their effect on the clearance of LC tissular deposits, although possibly accepted, remains to be demonstrated. Another study reported the disappearance of nodular mesangial lesions and disappearance of kappa light chain deposits in a patient with kidney LCDD under long-term chemotherapy [15].

\section{Conclusion}

These data indicate that chemotherapy, based on novel anti-myeloma agents, should be rapidly considered in LCDD patients with severe organ involvement, in order to rapidly induce efficient and sustained suppression of the pathogenic monoclonal LC.

Our case confirms that liver involvement may be a important complication that requires prompt diagnosis and therapy.

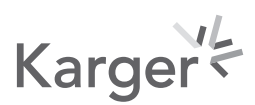




\section{Case Reports in Oncology}

\begin{tabular}{l|l}
\hline Case Rep Oncol 2020;13:1343-1348 \\
\hline DOI: 10.1159/000509508 & $\begin{array}{l}\text { @ 2020 The Author(s). Published by S. Karger AG, Basel } \\
\text { www.karger.com/cro }\end{array}$ \\
\hline
\end{tabular}

Grembiale et al.: A Cholestatic Hepatitis due to LCDD

\section{Statement of Ethics}

The patient has given his written informed consent to publish his case including publication of images.

\section{Conflict of Interest Statement}

The authors have no conflicts of interest to declare.

\section{Funding Sources}

None.

\section{Author Contributions}

A. Grembiale: acquisition, analysis, and interpretation of data for the work; drafting the work or revising it critically for important intellectual content; final approval of the version to be published. E. Garlatti, A. Ermacora: Substantial contributions to the conception and interpretation of data for the work; drafting the work or revising it critically for important intellectual content, final approval of the version to be published. S. Grazioli, M. Balbi, M. Tonizzo: revising the work critically for important intellectual content and final approval of the version to be published.

\section{References}

1 Randall RE, Williamson WC Jr, Mullinax F, Tung MY, Still WJ. Manifestations of systemic light chain deposition. Am J Med. 1976;60(2):293-9.

2 Buxbaum JN, Chuba JV, Hellman GC, Solomon A, Gallo GR. Monoclonal immunoglobulin deposition disease: light chain and light and heavy chain deposition diseases and their relation to light chain amyloidosis. Clinical features, immunopathology, and molecular analysis. Ann Intern Med. 1990;112(6):455-64.

3 Pozzi C, D'Amico M, Fogazzi GB, Curioni S, Ferrario F, Pasquali S, et al. Light chain deposition disease with renal involvement: clinical characteristics and prognostic factors. Am J Kidney Dis. 2003 Dec;42(6):1154-63.

4 Girelli CM, Lodi G, Rocca F. Kappa light chain deposition disease of the liver. Eur J Gastroenterol Hepatol. 1998; 10(5):429-30.

5 Droz D, Noel LH, Carnot F, Degos F, Ganeval D, Grunfeld JP. Liver involvement in nonamyloid light chain deposits disease. Lab Invest. 1984;50(6):683-9.

6 Said S, J Cooper C, C Nwosu A, E Bilbao J, T Hernandez G. Hypertension, renal failure, and edema in a 38-year-old man: light chain deposition disease; a case report and review of the literature. J Nephropathol. 2014;3(2): 63-8.

7 Weisel KC, Böckeler M, Bianchi L, Terracciano LM, Mayer F, Kanz L. Development of rapid light-chain deposition disease in hepatic arteries with severe ischemic cholangitis in a multiple myeloma patient treated with melphalan, prednisone and lenalidomide. Int J Hematol. 2009;89(1):91-4.

8 Michopoulos S, Petraki K, Petraki C, Dimopoulos MA. Light chain deposition disease of the liver without renal involvement in a patient with multiple myeloma related to liver failure and rapid fatal outcome. Dig Dis Sci. 2002;47(4):730-4.

9 Leung N, Bridoux F, Hutchison CA, Nasr SH, Cockwell P, Fermand JP, et al. International Kidney and Monoclonal Gammopathy Research Group. Monoclonal gammopathy of renal significance: when MGUS is no longer undetermined or insignificant. Blood. 2012 Nov 22;120(22):4292-5.

10 Jimenez-Zepeda VH. Light chain deposition disease: novel biological insights and treatment advances. Int J Lab Hematol. 2012;34(4):347-55.

11 Mena-Durán A, Muñoz Vicente E, Pareja Llorens G, Sanchis Cervera J. Liver failure caused by light chain deposition disease associated with multiple myeloma. Intern Med. 2012;51(7):773-6. 
12 Pozzi C, Locatelli F. Kidney and liver involvement in monoclonal light chain disorders. Semin Nephrol. 2002; 22(4):319-30.

13 Wada Y, Iyoda M, Saito T, Arai-Nunota N, Iseri K, Tomita E, et al. Light-chain deposition disease successfully treated with bortezomib in an elderly patient: a case report and review of the literature. Intern Med. 2015; 54(22):2893-8.

14 Palladini G, Sachchithanantham S, Milani P, Gillmore J, Foli A, Lachmann H, et al. A European collaborative study of cyclophosphamide, bortezomib, and dexamethasone in upfront treatment of systemic AL amyloidosis. Blood. 2015 Jul 30;126(5):612-5.

15 Cohen C, Royer B, Javaugue V, Szalat R, El Karoui K, Caulier A, et al. Bortezomib produces high hematological response rates with prolonged renal survival in monoclonal immunoglobulin deposition disease. Kidney Int. 2015 Nov;88(5):1135-43. 